\title{
EFFECT OF COOLING RATE ON MICROSTRUCTURE OF B2-NISC INTERMETALLICS
}

\author{
Zhipeng Yuan - Hao Chen* - Hongbao Cui - Yatao Chang - Xuefeng Guo
}

Materials Science \& Engineering School, Henan Polytechnic University, Jiaozuo 454000, The People's Republic of China

\begin{tabular}{l}
\hline ARTICLE INFO \\
\hline Article history: \\
Received: 22.12 .2016$. \\
Received in revised form: 13.10 .2017$. \\
Accepted: 13.10 .2017$. \\
\hline Keywords: \\
Centrifugal casting \\
Microstructure evolution \\
Rapid solidification \\
Heat treatment \\
(Ni $i_{2}$ Sc + NiSc) eutectic \\
\hline DOI: http://doi.org/10.30765/er.39.3.04
\end{tabular}

\section{Introduction}

Due to an excellent high temperature resistance, wear resistance, oxidation resistance and other excellent performance, intermetallics are widely applied in various fields, such as petrochemical, aerospace, and

\begin{abstract}
:
$\mathrm{Ni-50at \% Sc}$ alloy was prepared by centrifugal casting method. Volume fraction, the actual content of B2-NiSc and second phase Ni2Sc in alloy were analyzed with an Image-Pro Plus software. The cooling rates for the solidified thin plate with thickness of $2.65 \mathrm{~mm}, 1.2 \mathrm{~mm}, 0.75 \mathrm{~mm}$ and $0.35 \mathrm{~mm}$ are $1164,2570,4112$ and $8811 \mathrm{~K} \cdot \mathrm{s}$ 1 , respectively. It is found that $d=0.5 \mathrm{~mm}$ was an critical dimension which corresponds to an abrupt change in the solidification rate. It is also found that $(\mathrm{Ni2Sc}+\mathrm{NiSc})_{\text {eutectic }}$ was dispersed at grain boundary or between dendritic arms due to the loss of Sc element during melting. While $d>0.5 \mathrm{~mm}$ (corresponding to the thin plate with thickness of $0.75 \mathrm{~mm}, 1.20 \mathrm{~mm}$ and $2.65 \mathrm{~mm}$ ), the solidification structure consists of primary phase B2-NiSc and $(\mathrm{Ni2Sc}+\mathrm{NiSc})_{\text {eutectic. While }} d<0.5 \mathrm{~mm}$ (corresponding to the thin plate with thickness of $0.35 \mathrm{~mm}$ ), the solidification structure is composed of fine globular B2-NiSc and relatively small amounts of $(\mathrm{Ni2Sc}+\mathrm{NiSc})_{\text {eutectic. Based on the phase }}$ volumetric analyzing of the microstructure with an Image-Pro Plus software, the loss of Sc element during melting was about 3.01 3.10 at\%. The eutectic NiSc in the lamellar eutectic structure together with the primary phase B2-NiSc form a larger single phase NiSc, while Ni2Sc with the form of particles is distributed on the grain boundaries after $\left(970{ }^{\circ} \mathrm{C}, \quad 72\right.$ h) homogenization heat treatment.
\end{abstract}

energy power industry [1]. However, the fatal weakness of intermetallics is a low fracture toughness, which severely limits its application. To overcome the shortage, numerous experiments are carried out. In 2003, Gschneidner K. [2] reports that $\mathrm{RM}$ intermetallics with the B2 structure (where R

\footnotetext{
* Corresponding author. Tel.: +86 03913987124

E-mail address: chenhao@hpu.edu.cn.
} 
denotes a rare-earth element and $\mathrm{M}$ refers to a transition element or main group element) of such as $\mathrm{YRh}, \mathrm{DyCu}, \mathrm{CeAg}$ and $\mathrm{YAg}$ have high intrinsic plasticity. The intrinsic plasticity of these intermetallics was examined by a tensile test. YAg elongated $27 \%$ before breaking, DyCu elongated $16 \%$, and $\mathrm{YCu}$ elongated $11 \%$. These values are significantly different from the traditional concept of intrinsic ductility and toughness [3-6]. High ductility of the RM intermetallics has attracted more and more attention of scientists.

$\mathrm{NiSc}$ has been found to have the B2-CsCl type structures, and crystallized in the $\mathrm{Pm}-3 \mathrm{~m}$ space group. Referring to the previous studies, most of the research focus on the thermodynamic properties of the Ni-Sc two element system, and few studies on its intrinsic properties are carried out [7-10]. Therefore, the intrinsic plasticity of NiSc is of great significance, and it also has a significant reference for the followup research. In order to reveal the characteristics of the B2 intrinsic plasticity, it is extremely necessary to prepare $\mathrm{B} 2$ intermetallics without the second phase. However, the B2-NiSc intermetallics without any second phase are difficult to achieve due to the loss of Sc element during melting. It is certain that the formation, distribution, quantity and nature of the second phase are closely related to properties of B2$\mathrm{NiSc}$ intermetallics. Therefore, in this paper, characteristics of the B2-NiSc intermetallics were studied by rapid solidification technique.

In addition, according to the relationship between the vacancy in the crystal and temperature [11], $n_{\mathrm{v}}=v \cdot \exp \left[Q_{\mathrm{v}} / R T\right]$, the number of vacancy in the unit area increases with the increase of the temperature. By rapid solidification, the vacancies

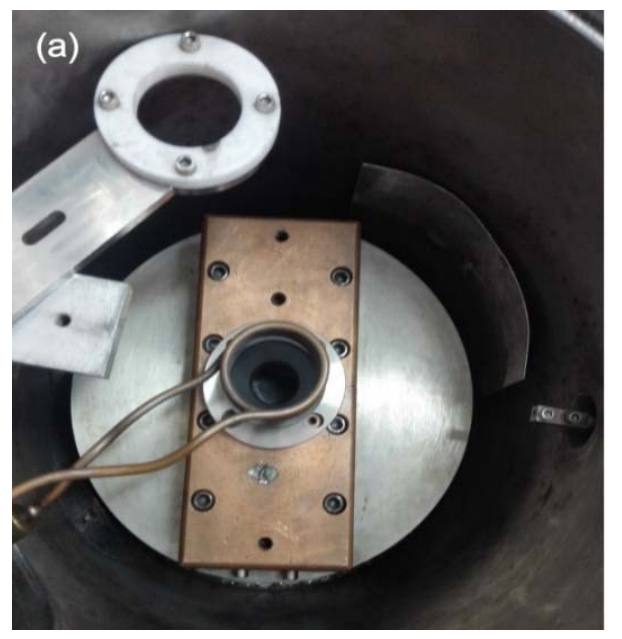

are fixed and it is possible to obtain a more homogeneous B2 intermetallics, which can reveal its intrinsic performance more realistically. Therefore, rapid solidification technology was used to study the microstructure evolution of the B2-NiSc intermetallics and the influence of rapid solidification on microstructure was analyzed.

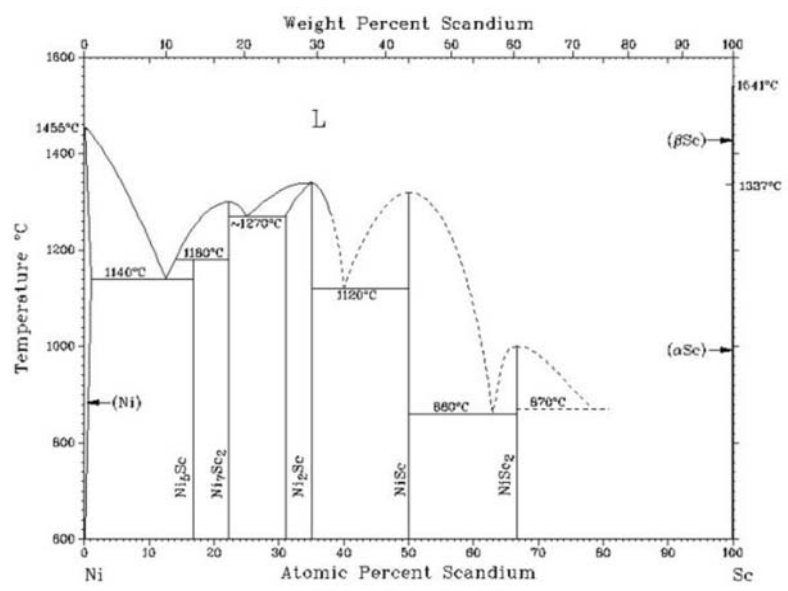

Figure 1. Binary phase diagram of Ni-Sc alloy.

\section{Experimental investigation}

The alloy with a nominal composition of $\mathrm{Ni}-50 \% \mathrm{Sc}$ (at.\%) was prepared by arc melting of a mixture of high purity elements of nickels(99.99 wt.\%) and $\mathrm{Sc}(99.99 \mathrm{wt} \%)$ under titanium gettered argon atmosphere. They were melted four times in order to achieve the chemical homogenization. The thin plates of $0.4 \mathrm{~mm}, 0.7 \mathrm{~mm}, 1.2 \mathrm{~mm}$ and $2.65 \mathrm{~mm}$ were obtained by centrifugal casting method. Fig. 2 shows centrifugal casting equipment.

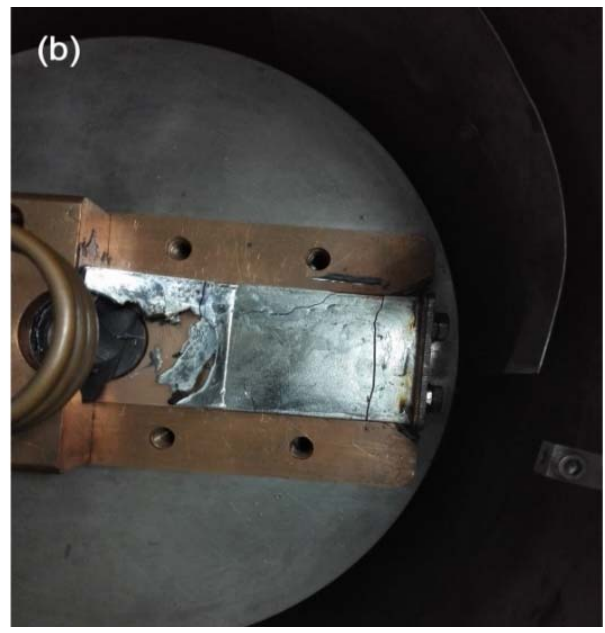

Figure 2. Centrifugal casting equipment. 
After the finished experiment, the samples were polished and etched with a solution of $1 \mathrm{ml} \mathrm{HF}+4 \mathrm{ml}$ $\mathrm{HNO}_{3}+5 \mathrm{ml} \mathrm{H}_{2} \mathrm{O}$ to examine the microstructure. The optical microscope (MA) and the scanning electron microscope (SEM) were used to examine the microstructure. The SEM investigations were carried out in scanning electron microscope operated at 20 $\mathrm{KV}$ and linked with an energy dispersive spectrometry (EDS) attachment. Chemical composition was determined by energy dispersion Xray spectroscope (EDS). The XRD measurements were carried out in X-ray diffractometer in the $2 \theta$ range from $20^{\circ}$ to $120^{\circ}$.

\section{Results and discussion}

\subsection{Microstructure and composition of $\mathrm{Ni}-50 \% \mathrm{Sc}$ by rapid solidification}

Binary phase diagram of Ni-Sc alloy is shown in Fig. 2. When the composition is $\mathrm{Ni}-50 \% \mathrm{Sc}$ (mole fraction), the NiSc intermetallics with the B2 structure can be obtained. When the content of $\mathrm{Sc}$ is lower than $50 \% \mathrm{Sc}$, the $\mathrm{NiSc}_{\text {primary }}$ and $\left(\mathrm{Ni}_{2} \mathrm{Sc}+\mathrm{NiSc}\right)_{\text {eutectic }}$ are obtained; On the contrary, when the content of $\mathrm{Sc}$ is higher than $50 \% \mathrm{Sc}$, the $\mathrm{NiSc}_{\text {primary }}$ and $\left(\mathrm{NiSc}+\mathrm{NiSc}_{2}\right)_{\text {eutectic }}$ are obtained. B2$\mathrm{NiSc}$ is a linear intermetallics. Thus, under the condition of equilibrium or near equilibrium solidification, no matter how high the purity of the metal is, a homogeneous single-phase intermetallics cannot be obtained. In addition, Sc, a silver-white transition metal, usually quickly oxidized in the air and with a lost luster. Thus, the loss of $\mathrm{Sc}$ is unavoidable during the smelting process, solidification of B2-NiSc usually accompanies the appearance of the second phase.

Fig. 3 represents central region microstructures of Ni$50 \% \mathrm{Sc}$ alloy by centrifugal casting with a $2.65 \mathrm{~mm}$ thickness. The primary phase is the B2-NiSc intermetallics and the microstructure between dendrite arm and dendrite is lamellar eutectic structure, as shown in Fig. 3(a) and Fig. 3(b), respectively.

The XRD analysis results of NiSc alloy is shown in Fig. 4. The XRD analysis indicates that microstructures of the alloy is composed of B2-NiSc and $\mathrm{Ni}_{2} \mathrm{Sc}$. The EDS analysis shows that the dendritic composition is $\mathrm{Ni}-50.79 \% \mathrm{Sc}$, which is $\mathrm{B} 2-\mathrm{NiSc}$ linear intermetallics. The eutectic composition is $\mathrm{Ni}$ $38.93 \% \mathrm{Sc}$, which is $\left(\mathrm{Ni}_{2} \mathrm{Sc}+\mathrm{NiSc}\right)$ eutectic mixture. From the above analysis, it can be known that the nominal composition of Ni- $50 \% \mathrm{Sc}$ cannot obtain $100 \%$
B2-NiSc intermetallics by centrifugal casting method. Due to the loss of Sc during the experiment, the actual composition was shifted to the left side of the linear B2-NiSc intermetallics. Thus, obtaining the 100\% B2-NiSc intermetallics is entirely a small probability event.

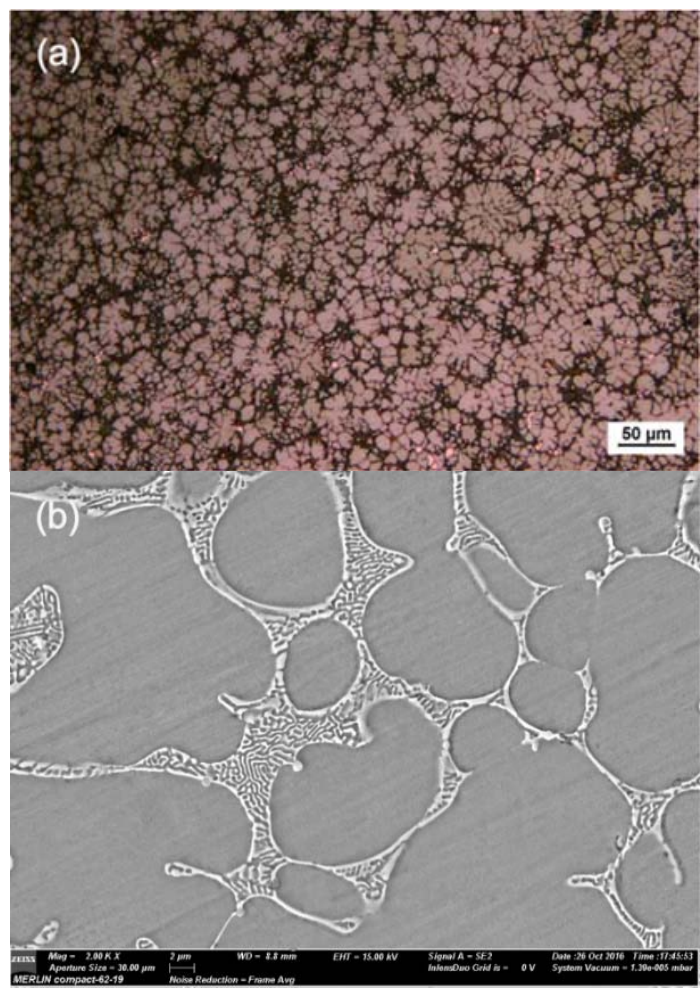

Figure 3. Microstructures of Ni-50\%Sc alloy by Centrifugal Casting method with $2.65 \mathrm{~mm}$ thickness: (a) Microstructure; (b) Eutectic microstructure between dendrites.

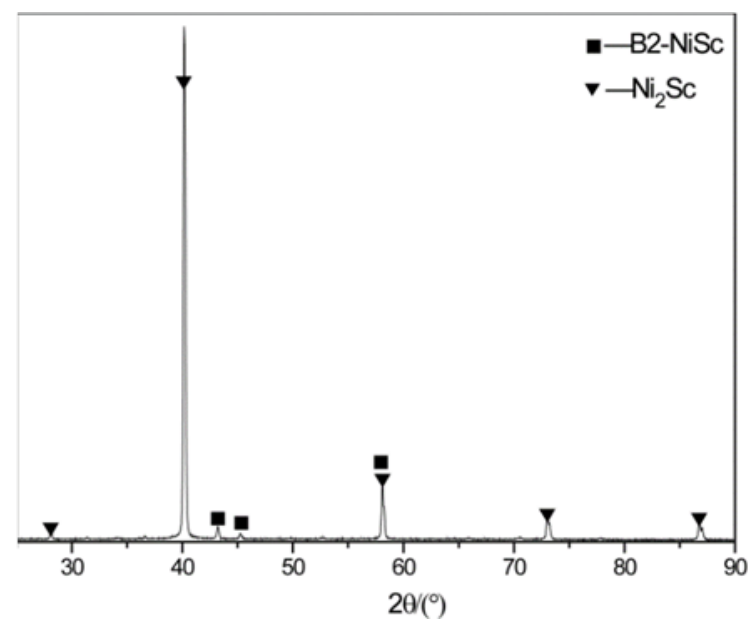

Figure 4.XRD pattern of Ni-50\%Sc alloy by the Centrifugal Casting method with $2.65 \mathrm{~mm}$ thickness. 


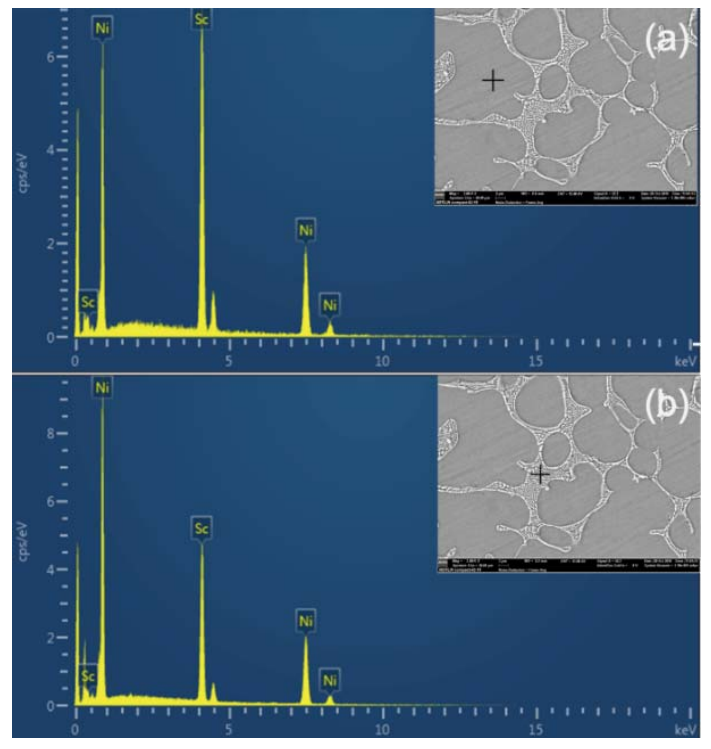

Figure 5. The EDS results of Ni-50\%Sc alloy by the Centrifugal Casting method with $2.65 \mathrm{~mm}$ thickness: (a) Primary phase; (b) Eutectic microstructure.

Volume fraction and the actual content of the primary phase B2-NiSc and $\left(\mathrm{Ni}_{2} \mathrm{Sc}+\mathrm{NiSc}\right)_{\text {eutectic in } \mathrm{Ni}-50 \% \mathrm{Sc}}$ were analyzed with an Image-Pro Plus software. By using lever rule, the proportion of each element in the sample is calculated, and the amount of the Sc loss in the experiment can be further obtained. Based on the phase volumetric analyzing of the microstructure with an Image-Pro Plus software, the loss of Sc element during melting is about $3.01 \% \sim 3.10 \%$.

\subsection{Effect of cooling rate on the solidification structure of $\mathrm{Ni}-50 \% \mathrm{Sc}$ alloy}

Fig.6 shows central region microstructures of Ni$50 \% \mathrm{Sc}$ alloy of four different thickness by centrifugal casting method. It can be seen from Fig. 6 that the smaller the thickness, the stronger the quenching capacity, the finer the microstructure and the less eutectic structure.

The thickness of thin plate is not affected by the type and microstructures of the solidified alloy. Thus, the thickness of the thin plate which was prepared by the centrifugal casting method and cooling rate have the following relationship [12].

$$
R=\frac{h\left(T_{\mathrm{i}}-T_{0}\right)}{\left(C_{\mathrm{P}} \cdot \rho \cdot t\right)}
$$

where, $h$ is the thermal diffusivity of the melt alloy and the rotating roll; $T_{\mathrm{i}}$ and $T_{0}$ are the temperatures of the melt alloy and the rotating roll, respectively; $C_{\mathrm{p}}$ is the specific heat of the melt alloy; $\rho$ is the density of the melt alloy; $t$ is the thickness of the thinplate by rapidly solidified method.
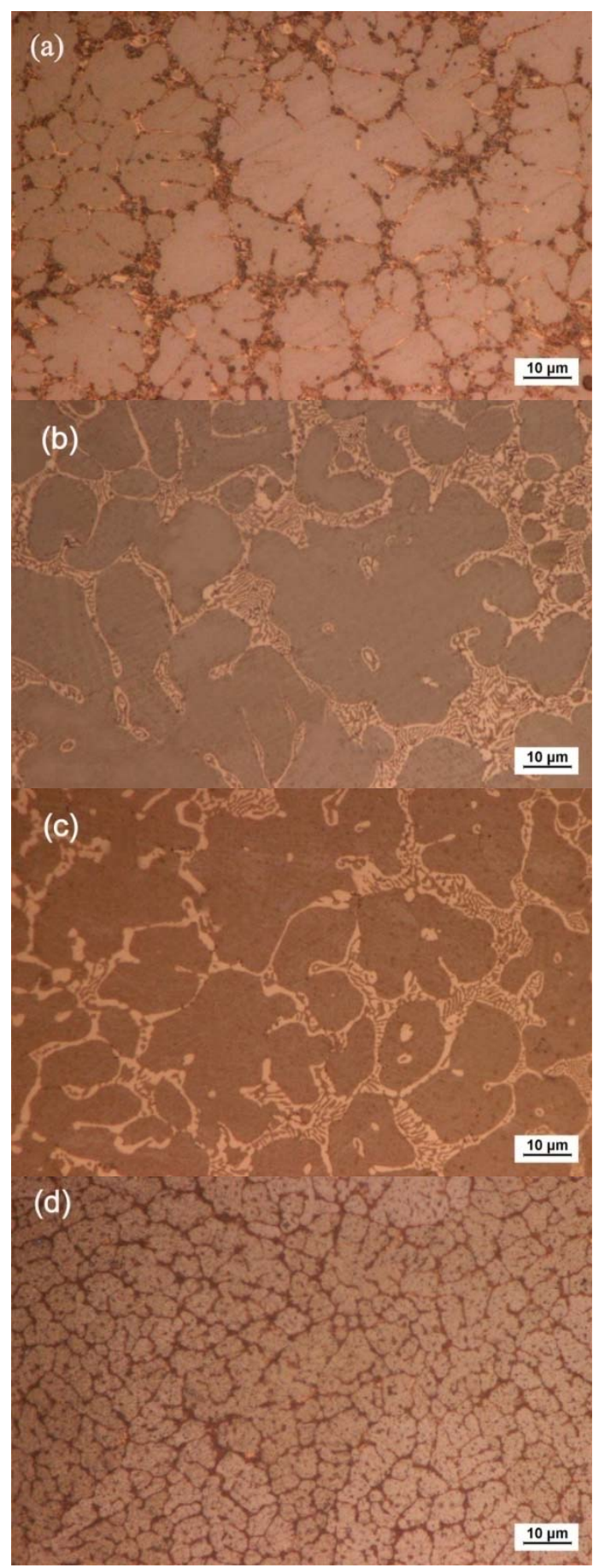

Figure 6. Microstructures of Ni-50\%Sc alloy by centrifugal casting method with different thickness: (a) $2.65 \mathrm{~mm}$; (b) $1.2 \mathrm{~mm}$; (c) 0.75 $\mathrm{mm}$; (d) $0.35 \mathrm{~mm}$. 
The solidification structure of the alloy is not only related to the composition of the alloy, but also to the solidification condition, such as the cooling rate. When the alloys have the same composition, the cooling rate plays an important role in the formation of solidified structure.

For copper rolls, $h=1.0 \mathrm{cal} /\left(\mathrm{cm}^{2} \cdot K \cdot \mathrm{s}\right)$ [13]. Density $\rho$ and specific heat $C_{\mathrm{p}}$ of $\mathrm{Ni}-50 \% \mathrm{Sc}$ alloy are $5.398 \mathrm{~g} / \mathrm{cm}^{3}$ and $0.29 \mathrm{~J}(\mathrm{~g} \cdot \mathrm{K})$, respectively. $T_{\mathrm{i}}=1453 \mathrm{~K}, T_{0}=298 \mathrm{~K}, T_{\mathrm{i}}-T_{0}=1155 \mathrm{~K}$, according to the binary phase diagram of NiSc alloy.

$$
R=3084 / t
$$

The results calculated by equation (2) are shown in Fig. 7. The cooling rate increases with the decrease of the thickness of the thin plate, which indicates that the thickness of the thin plate has a great influence on the cooling rate. It can be seen from Fig.7 that the solidification rate changes little when the thickness of the thin plate decreases from $2.6 \mathrm{~mm}$ to $1.2 \mathrm{~mm}$. When the thickness of the thin plate decreases from $1.2 \mathrm{~mm}$ to $0.35 \mathrm{~mm}$, cooling rate changes a lot. The cooling rate of the $0.35 \mathrm{~mm}$ thinplate was 7.43 times as large as the $2.65 \mathrm{~mm}$ thin plate.

$$
R=3084 / t^{2}
$$

The relationship between the variation of cooling rate obtained from equation (3) and the thickness of the thin plate is shown in Fig. 7. When the thickness of the thin plate is $0.35 \mathrm{~mm}$, the solidification rate increases rapidly and the cooling rate is high. As can be seen from Fig. 6 that the secondary dendritic arms of the dendrites are quite coarse when the thickness of the thin plate is $2.65 \mathrm{~mm}, 1.2 \mathrm{~mm}$ and $0.75 \mathrm{~mm}$. When the thickness of the thin plate is $0.35 \mathrm{~mm}$, the secondary dendritic growth is restrained because of the large cooling rate. The secondary dendrite arms of the dendrites are very short and the dendrites in the microstructure are basically spherical particles.

\subsection{Microstructure of Ni-50\%Sc after homogenization heat treatment}

Fig. 8 shows central region microstructures of Ni$50 \% \mathrm{Sc}$ alloy after homogenization heat treatment by centrifugal casting method. As can be seen from Fig. 8 that the primary phase $\mathrm{B} 2-\mathrm{NiSc}$ dendrite disappeared after $970{ }^{\circ} \mathrm{C}$ heat treatment for 72 hours. The eutectic NiSc in the lamellar eutectic structure, along with the primary phase B2-NiSc form a larger single phase, while $\mathrm{Ni}_{2} \mathrm{Sc}$ presents in the form of particles and distributes on the grain boundaries. The EDS analysis showed that the matrix is the B2-NiSc phase, irregular spherical particles or long Strip particle are $\mathrm{Ni}_{2} \mathrm{Sc}$ phase.
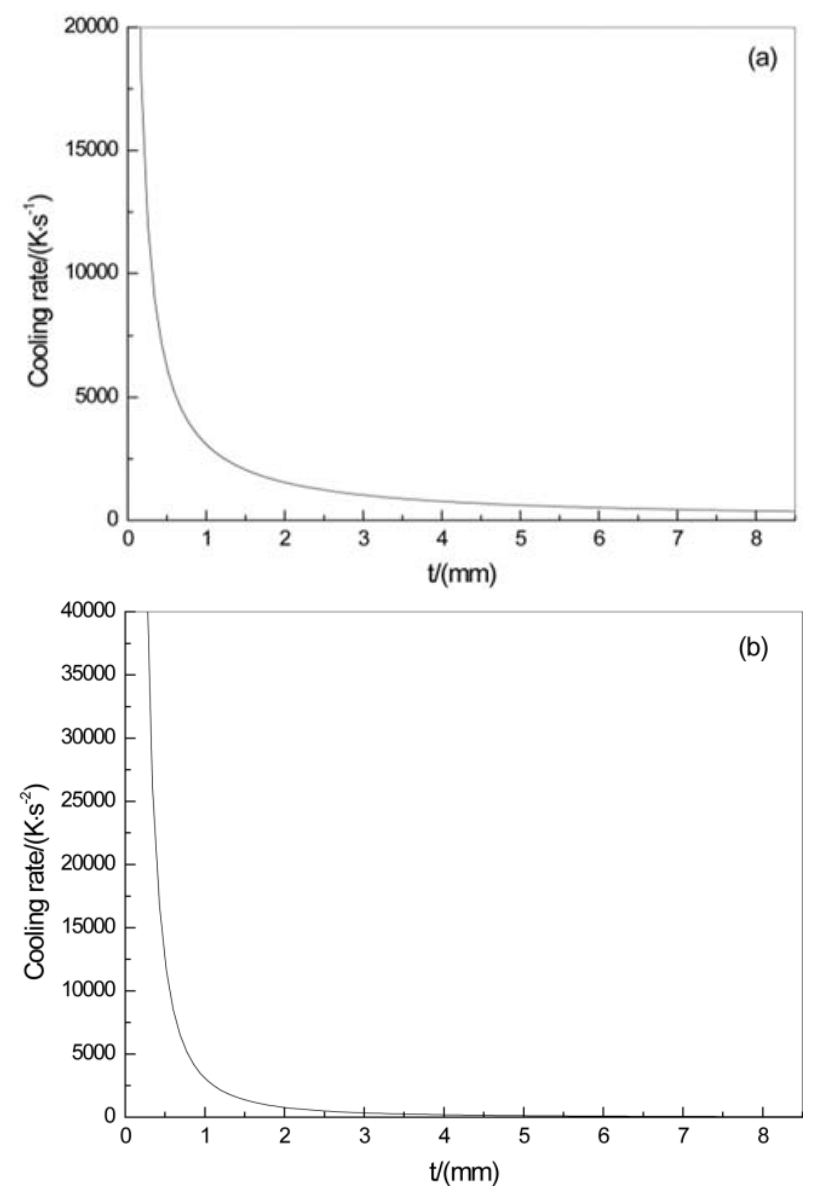

Figure7. (a) Relationships between cooling rate and thickness of specimens; (b) Relationships between variation of cooling rate and thickness of specimens.

Since the ratio of the surface area to the volume of the lamellar structure is larger than the ratio of the surface area to the volume of the spherical structure, the lamellar structure has a larger surface energy than the spherical structure. Thus, the lamellar structure has a tendency to spontaneously change into spherical particles. This spontaneous process is based on diffusion. The diffusion of atoms in the alloy occurs after a long time of heat treatment. The eutectic NiSc in the lamellar eutectic structure together with the primary phase $\mathrm{B} 2-\mathrm{NiSc}$ form a larger single phase crystal, while $\mathrm{Ni}_{2} \mathrm{Sc}$ with the form of particles is distributed on the grain boundaries. 


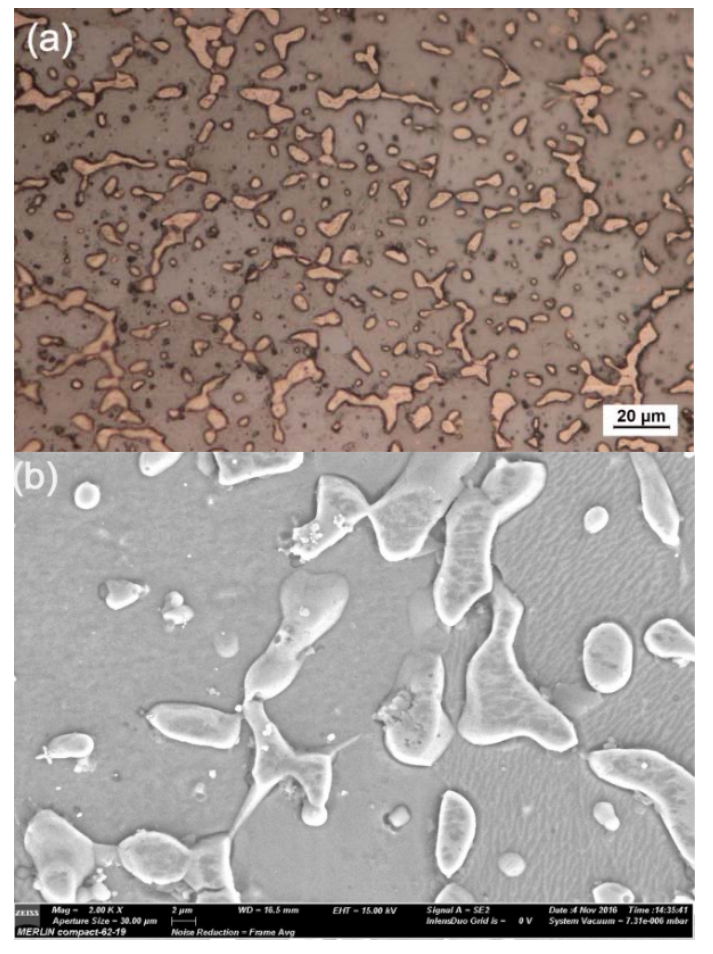

Figure 8. Central region microstructures of $\mathrm{Ni}$ $50 \%$ Sc alloy after homogenization heat treatment by centrifugal casting method with $2.65 \mathrm{~mm}$ thickness.
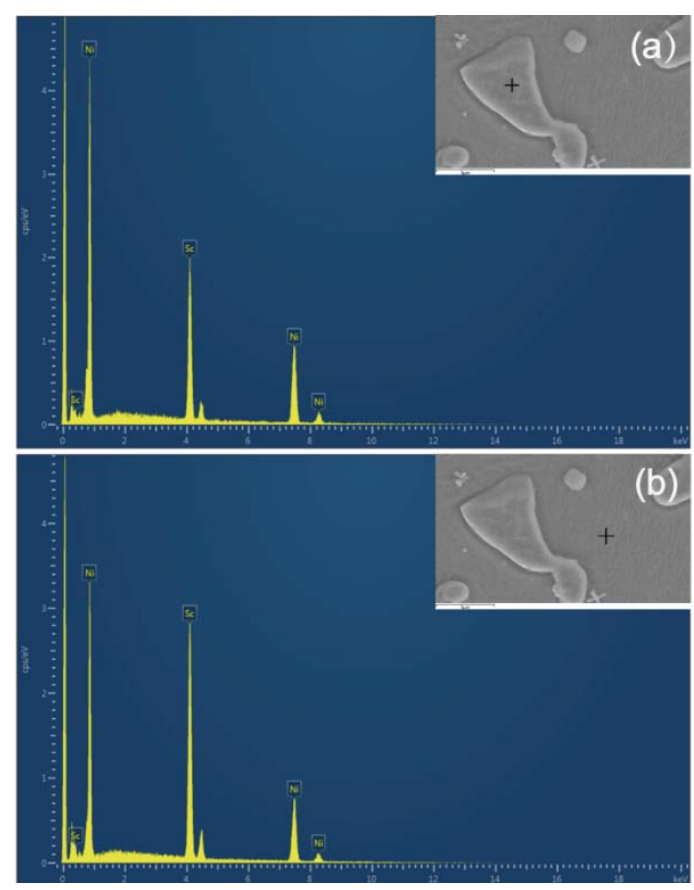

Figure 9. The EDS results of the Ni-50\%Sc alloy after homogenization heat treatment by the centrifugal casting method $w 2.65 \mathrm{~mm}$ thickness: (a) spherical particle; (b) matrix.

\section{Conclusion}

(1) It is also found that $\left(\mathrm{Ni}_{2} \mathrm{Sc}+\mathrm{NiSc}\right)_{\text {eutectic }}$ is dispersed at grain boundary or between dendritic arms due to the loss of Sc element during melting. Based on the phase volumetric analyzing of the microstructure with an Image-Pro Plus software, the loss of Sc element during melting is about $3.01 \% \sim 3.10 \%$ in accordance to specimens thickness. (2) Thickness of the thin plate has a great influence on the structure. While $d>0.5 \mathrm{~mm}$ (corresponding to the specimens with thickness of $0.75 \mathrm{~mm}, 1.2 \mathrm{~mm}$ and $2.65 \mathrm{~mm}$ ), the solidification structure consists of the primary phase $\mathrm{B} 2-\mathrm{NiSc}$ and $\left(\mathrm{Ni}_{2} \mathrm{Sc}+\mathrm{NiSc}\right)_{\text {eutectic. }}$ While $d<0.5 \mathrm{~mm}$ (corresponding to the specimens with thickness of $0.35 \mathrm{~mm}$ ), the solidification structure consists of fine globular B2-NiSc and relatively small amounts of $\left(\mathrm{Ni}_{2} \mathrm{Sc}+\mathrm{NiSc}\right)_{\text {eutectic }}$.

(3) The primary phase B2-NiSc dendrite disappeared after $\left(970{ }^{\circ} \mathrm{C}, 72 \mathrm{~h}\right)$ homogenization heat treatment. The eutectic NiSc in the lamellar eutectic structure together with the primary phase B2-NiSc form a larger single phase crystal, while $\mathrm{Ni}_{2} \mathrm{Sc}$ in the form of particles is distributed on the grain boundaries.

\section{Acknowledgements}

This work is supported by the Natural Science Foundation of China (NSFC) under the Grant No. 51301063 and No. 51271073, Talent Introduction Foundation of Henan Polytechnic University (Y2009) and Science and technology research key project guidance plan for Henan province education department(12B430008).

\section{References}

[1] Cao, G. H., Tian, H. N., Liu, N., Li, X., Ren, Z. M.: Mechanical properties of the B2 NdAg intermetallic compound, Scripta Materialia, 65 (2011), 2, 147-150.

[2] Jr, G. K., Russell, A., Pecharsky, A., Morris, J., Zhang, Z.: A family of ductile intermetallic compounds, Nature Materials, 2 (2003), 9, 587590.

[3] Mulay, R. P., Wollmershauser, J. A., Heisel, M. A., Bei, H., Russell, A. M.: X-ray diffraction study of the phase purity, order and texture, of ductile B2 intermetallics, Acta materialia, 58 (2010), 7, 2788-2796.

[4] Russell, A. M., Zhang, Z., Gschneidner, K. A., Lograsso, T. A., Slager, A. J.: Mechanical 
properties of single crystal YCu and $\left(T b_{0.88} D y_{0.12}\right) Z n$ B2 intermetallic compounds, Intermetallics, 13 (2005), 6, 565-571.

[5] Cao, G. H., Becker, A. T., Wu, D., Chumbley, L. S., Lograsso, T. A.: Mechanical properties and determination of slip systems of the B2 YZn intermetallic compound, Acta Materialia, 58 (2010), 12, 4298-4304.

[6] Zhang, Z., Russell, A. M., Biner, S. B., Gschneidner, K. A., Lo, C. C. H.: Fracture toughness of polycrystalline $\mathrm{YCu}, \mathrm{DyCu}$, and YAg, Intermetallics, 13 (2005), 5, 559-564.

[7] Cao, Z. P., Liu, S. H., Xu, F., Cheng, K. M., Gao, Q. N.: Experimental investigation and thermodynamic analysis of the Sc-Ni system supplemented with first-principles calculations, Thermochimica Acta, 586 (2014), 8, 30-39.

[8] Shevchenko, M. A., Ivanov, M. I., Berezutskii, V. V., Kudin, V. G., Sudavtsovae, V. S.: Thermodynamic properties of alloys of the $\mathrm{Ni}-\mathrm{Sc}$ and Ni-Y systems, Russian Journal of Physical Chemistry, 88 (2014), 6, 897-902.
[9] Zhu, D. M., Li, C. R., Guo, C. P., Du, Z. M., Li, J. Q.: Thermodynamic assessment of the $\mathrm{Ni}-\mathrm{Sc}$ binary system, Calphad-computer Coupling of Phase Diagrams \& Thermochemistry, 48 (2015), 3, 106-112.

[10] Kardellass, S., Servant, C., Selhaoui, N., Iddaoudi, A., Amar, M. A.: Thermodynamic description of the $\mathrm{Ni}-\mathrm{Sc}$ system, Calphadcomputer Coupling of Phase Diagrams \& Thermochemistry, 42 (2013), 42, 59-65.

[11] Askeland, D. R., Fulay, P. P.: Essentials of materials science and engineering, Cengage Learning, American, 2013.

[12] Meng, X., Gao, S., Ma, L., Li, Z., Yue, Y.: Effects of rotational velocity on microstructures and mechanical properties of surface compensation friction stir welded 6005A-T6 aluminum alloy, Engineering Review, 35 (2016), 4, 375-379.

[13] Cao, M. S.: Physical Metallurgy Foundation, Metallurgical industry Press, 1985. 